\title{
Radio Continuum Maps of Southern Barred Spiral Galaxies
}

\author{
Margaret Mazzolini, Janice Long and Rachel Webster \\ Astrophysics Group, School of Physics, University of Melbourne, \\ Parkville VIC 3052
}

\begin{abstract}
A sample of southern spiral galaxies are being observed at 20 and $13 \mathrm{~cm}$ with the Australia Telescope Compact Array in order to look for examples of anomalous outflows from the nuclear region. In this paper we discuss the radio continuum maps of the three barred spiral galaxies observed to date.
\end{abstract}

\section{Introduction}

A number of spiral galaxies are known to exhibit anomalous non-thermal radio structures extending out of the plane from their nuclear regions, sometimes to a distance of several kiloparsecs. Their interpretation in the literature has reflected a difference of opinion: either these structures are attributed to the effect of circumnuclear starbursts or of activity within the galactic nucleus itself. We are studying a sample of southern spiral galaxies to find examples of these anomalous radio structures and to help determine the frequency of their occurrence.

\section{Observations}

The three barred galaxies discussed here have each been observed with two different $6 \mathrm{~km}$ array configurations at the Australia Telescope Compact Array (ATCA) in continuum mode at 13 and $20 \mathrm{~cm}$, with a $128 \mathrm{MHz}$ bandwidth. Total observation times are: 31.5 hours for NGC 3059, 25 hours for NGC 6300, and 23 hours for NGC 1097. The MIRIAD (Multichannel Image Reconstruction, Image Analysis and Display) Package was used to invert the data using firstly uniform weighting, for maximum resolution, then natural weighting for optimum signal-to-noise ratio. Only NGC 1097 is a sufficiently strong source to allow selfcalibration. The maps for NGC 1097 have been previously reported (Mazzolini \& Webster 1995) but are discussed here also for completeness.

The coordinates and classification of each galaxy are listed in the table together with the map noise levels and restoring beam FWHM dimensions for both observing wavelengths. 


\begin{tabular}{cccccc} 
Galaxy & $\begin{array}{c}\text { coordinates } \\
(\mathrm{B} 1950)\end{array}$ & type & $\begin{array}{c}\lambda \\
(\mathrm{cm})\end{array}$ & $\begin{array}{c}\text { map } \\
\text { noise } \\
(\mathrm{mJy} / \mathrm{bm})\end{array}$ & $\begin{array}{c}\text { restoring } \\
\text { beam } \\
(\operatorname{arcsec})\end{array}$ \\
\hline \multirow{2}{*}{ NGC 3059 } & $09: 49: 38,-73: 41: 12$ & $\mathrm{SB}(\mathrm{rs}) \mathrm{bc}$ & 20 & 0.2 & $9.2 \times 8.4$ \\
& & & 13 & 0.08 & $6.1 \times 5.4$ \\
NGC 1097 & $02: 44: 11,-30: 28: 54$ & $\mathrm{SB}(\mathrm{s}) \mathrm{b}$ & 20 & 0.2 & $11 \times 4.7$ \\
& & & 13 & 0.1 & $5.6 \times 2.7$ \\
NGC 6300 & $17: 12: 18,-62: 45: 54$ & $\mathrm{SB}(\mathrm{rs}) \mathrm{b}$ & 20 & 0.8 & $6.6 \times 5.0$ \\
& & & 13 & 0.4 & $3.6 \times 2.8$
\end{tabular}

\section{Discussion}

\subsection{NGC 3059}

NGC 3059 is a barred, ringed southern spiral galaxy viewed almost face on. It has previously been mapped in the radio at only very low resolution. The naturally-weighted 20 and $13 \mathrm{~cm}$ maps obtained in this study show extended radio emission in the disk of the galaxy. The galactic bar and the beginnings of the spiral arms are clearly visible, however data from a more compact array configuration is needed before the large-scale details of the maps can be considered reliable.

\subsection{NGC 1097}

NGC 1097 is a large, bright, southern barred galaxy with an active nucleus. Originally described as a LINER or Seyfert 2, the nucleus is recently (StorchiBergmann et al. 1993) displaying broad emission lines characteristic of a Seyfert 1. The galactic bar contains very linear dust lanes, and the galaxy has a nuclear ring composed of $\mathrm{H}$ II regions and hot-spots. Four spectacular optical jets extend to at least 4 galactic radii. $20 \mathrm{~cm}$, VLA radio-continuum observations ( $\mathrm{Hummel}$ et al. 1987, Wolstencroft et al. 1984) have revealed emission dominated by the nuclear ring including two hot-spots seen at other wavelengths, plus a bright unresolved nucleus. Other radio continuum observations at $1465 \mathrm{MHz}$ with the VLA (Ondrechen \& van der Hulst 1983) have revealed emission coincident with the linear dust lanes in the bar.

The 20 and $13 \mathrm{~cm}$ maps obtained so far in this study have been uniformlyweighted to maximize resolution. The nuclear ring and hot spots are well imaged at both 20 and $13 \mathrm{~cm}$. Our resolution is not good enough to detect the nucleus, but we do detect faint emission in the disk of the galaxy, which we hope to image more clearly once the $6 \mathrm{C}$ array data are analyzed. Because the optical character of the nucleus has been observed to change recently, once our second set of data is reduced we hope to obtain a reliable lower-limit flux estimate, in order to investigate whether the continuum radio flux has changed since the last VLA radio observations. 


\subsection{NGC 6300}

NGC 6300 is a large, barred and ringed southern spiral with a Seyfert 2 nucleus. $\mathrm{H} \alpha$ maps of NGC 6300 (Ryder \& Dopita 1993) show that "most of the starforming action seems to be taking place further out in a ring some 2.5 arcmin in diameter. Continuum subtraction indicates almost no star formation activity inside of this ring."

Our naturally-weighted maps have rather high map noise levels (see the table), reflecting the presence of confusing sources. In both cases the nucleus is well-fitted by a point-source; without data from a more compact ATCA configuration we would not expect to detect the 2.5 arcmin diameter ring seen by Ryder \& Dopita (1993). From our map we estimate that the total emitted flux from the nucleus, measured within contours set at 2.5 times the noise level, is $14 \mathrm{mJy}$ at $20 \mathrm{~cm}$ and $11 \mathrm{mJy}$ at $13 \mathrm{~cm}$.

A photometric and emission-line kinematics study of NGC 6300 by Buta (1987) found the rotation center of the galaxy to be approximately 5 arcsec east and probably slightly south of the optical nucleus. We obtain peak radio emission at about 2.4 arcsec to the east, but not significantly south, of the optical center.

Acknowledgments. This research has made use of the NASA/IPAC Extragalactic Database (NED) which is operated by the Jet Propulsion Laboratory, Caltech, under contract with the National Aeronautics and Space Administration. We wish to thank N. Killeen and B. Sault and the staff of the Australia Telescope National Facility for their assistance during the observing runs and also with the data reduction. M. M. M. acknowledges the support of a University of Melbourne Fellowship for Women with Career Interruptions.

\section{References}

Buta, R. 1987, ApJS, 64, 383

Hummel, E., van der Hulst, J. M., \& Keel, W. C. 1987, A\&A, 172, 32

Mazzolini, M. \& Webster, R. 1995, Pub. of the Astr. Soc. of Australia, in pres

Ondrechen, M. P. \& van der Hulst, J. M. 1983, ApJ, 269, L47

Ryder, S. \& Dopita, M. 1993, ApJS, 88, 415

Storchi-Bergmann, T., Baldwin, J. A., \& Wilson, A. S. 1993, ApJ, 410, L11

Wolstencroft, R. D., Perley, R., \& Tully, R. B. 1984, MNRAS, 207, 889 